\title{
KiteGen project: control as key technology for a quantum leap in wind energy generators
}

\author{
M. Canale, L. Fagiano, M. Milanese, M. Ippolito
}

\begin{abstract}
The paper investigates the control of tethered airfoils in order to devise a new class of wind generators, indicated as KiteGen, able to overcome the main limitations of the present aeolian technology based on wind mills. A model taken from the literature is used to simulate the dynamic of a kite whose lines are suitably pulled by a control unit. Energy is generated by a cycle composed of two phases, indicated as the traction and the drag one. The kite control unit is placed on the arm of a vertical axis rotor, which is connected to an electric drive able to act as generator when the kite lines pull the rotor and as motor in dragging the kite against the wind flow. In each phase, control is obtained by "fast" implementations of suitable NMPC designs. In the traction phase the control is designed such that the kite pulls the rotor arm, maximizing the amount of generated energy. When the kite is not able to generate energy any more, the control enters the drag phase and the kite is driven to a region where the energy spent to drag the rotor is a small fraction of the energy generated in the traction phase, until a new traction phase is undertaken. Simulation results are presented, showing that KiteGen may represent a quantum leap in wind energy generation.
\end{abstract}

\section{INTRODUCTION}

The solution of the problem posed by electric energy generation from fossil sources (high costs due to large demand increases in front of limited resources, pollution and $\mathrm{CO}_{2}$ production, geopolitical use of the fossil sources by the few producer countries) is an urgent and strategic issue of our society. It is evident that these problems can be overcome only with the use of sources which are renewable, cheap, easily available and sustainable for the environment. Actual renewable technologies have not such potentialities. Indeed, even the most optimistic forecast on the diffusion of present renewable sources (wind, photovoltaic, biomasses) estimates to reach a contribution to the world electric energy demand of no more than $20 \%$ within the next $15-20$ years. In particular, wind mills are currently the largest source of electric power produced with renewable energy (excluding hydro power plants) [1]. However, they require heavy towers, foundations and huge blades, which make a significant impact on the environment, require massive investments and long-term amortization periods. All these problems reflect in electric

Supported by Regione Piemonte under the Project "Controllo di aquiloni di potenza per la generazione eolica di energia" and by Ministero dell'Università e della Ricerca of Italy, under the National Project "Advanced control and identification techniques for innovative applications".

The first three authors are with Dipartimento di Automatica e Informatica, Politecnico di Torino, Italy. M. Milanese is also with Modelway, an academic spin-off society of Politecnico di Torino. M. ippolito is with Sequoia Automation, Chieri, Italy

e-mail: massimo.canale@polito.it, lorenzo.fagiano@polito.it, mario.milanese@polito.it,m.ippolito@sequoiaonline.com energy production costs that are not yet competitive, in strict economic sense, with the ones of thermal generators, despite recent large rises of oil and gas prices. Moreover, wind farms have wide problems of social acceptance due to their territory occupation, which is unacceptably higher than for thermal plants of the same power (up to 200-300 times). In order to overcome such limitations at Politecnico di Torino a new project has been started to design and build a new class of wind energy generators, indicated as KiteGen. The key idea (see the patents, [2], [3]) is to capture wind energy by means of tethered airfoils whose flight is suitably driven by an automatic control unit. It is expected that a wind generator of this type will have a territory occupation much lower than a wind farm of the same power (by a factor up to 50100 ) and much lower electric energy production costs (by a factor up to 10-20). In the first step of the KiteGen project a small scale prototype has been realized (see Fig. 1) to show the capability of controlling the flight of a single kite, by pulling the two lines which hold it, in such a way to extract a significant amount of energy. In [4] such a capability has

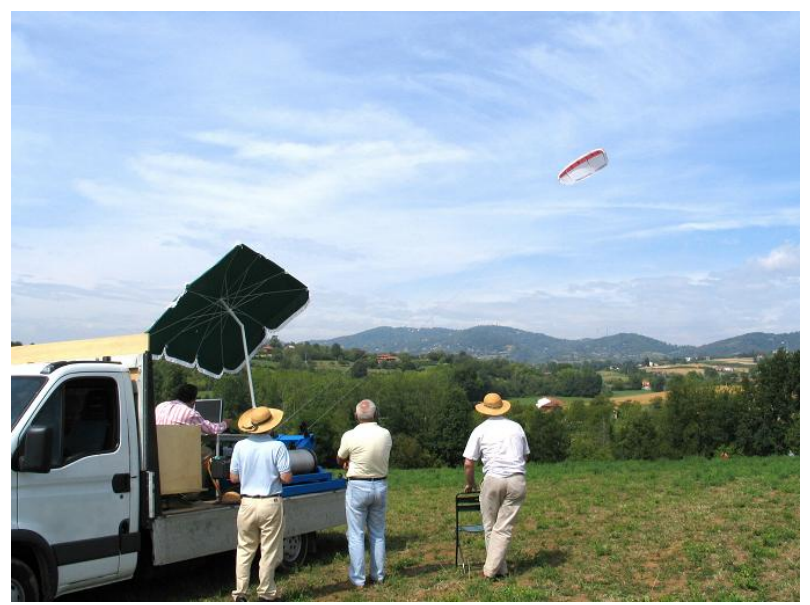

Fig. 1. KiteGen small scale prototype

been investigated in simulation, employing the kite model used in [5]. In the configuration considered in [4], indicated as "yo-yo" configuration, the Kite Steering Unit (KSU) (see fig. 2) has two electric actuators which act as motors in pulling the lines for controlling the flight or for recovering the kite and as generators if the lines length increases when pulled by the kite. The electric actuators which hold the lines are fixed with respect to the ground. Energy is generated by continuously repeating a cycle composed of two phases: 
the traction and the recovery ones. In the traction phase the control is designed such that the kite pulls the lines, so that a certain amount of energy is generated. When the maximal length of the lines is reached, the control enters into the recovery phase, where the kite is driven to a region where the lines can be pulled by the motors until the minimal length is reached, spending a small fraction of the energy generated in the traction phase, and a new traction phase is undertaken. The potentialities of the yo-yo configuration have also been investigated in [6] for the cases of one and two kites linked to a single cable: optimal kite periodic loops, which maximize the generated energy, are computed considering as inputs the derivatives of the kite roll angle and lift coefficient and of the cable winding speed. Another interesting application of power generating kites has been studied in [7], where optimal periodic loops are computed for a kite which is towing a ship. In this paper, the analysis of the KiteGen potentialities of energy generation will be presented, related to a carousel configuration.

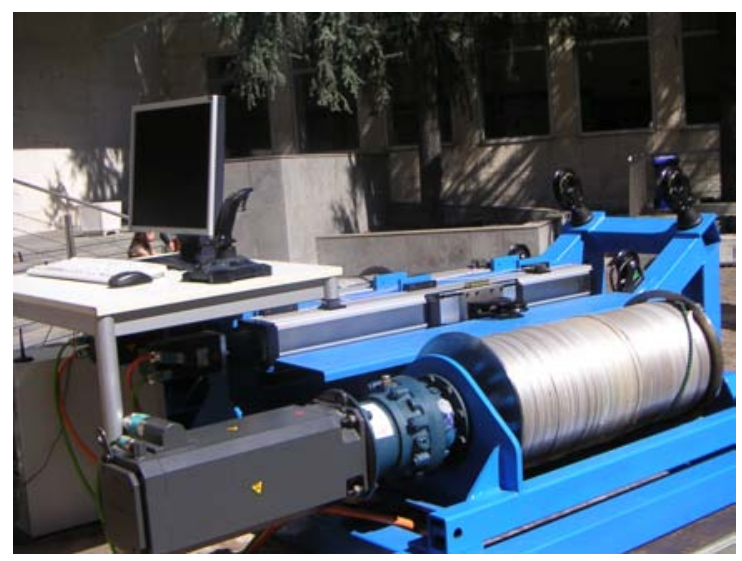

Fig. 2. Kite Steering Unit

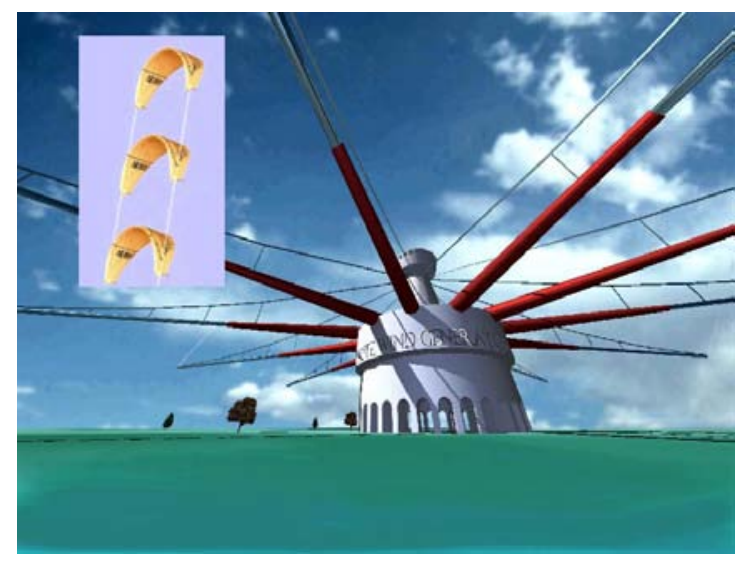

Fig. 3. Carousel configuration of KiteGen

In such configuration, several airfoils are controlled by their KSU placed on the arms of a vertical axis rotor (see Figure 3 ), and the control is designed to maximize the power transmitted by the airfoils to the rotor, suitably connected to an electric generator. The torque opposed to the motion by the electric generator is suitably controlled in order to keep the rotation speed constant. Energy is generated by continuously repeating a cycle composed of two phases, the traction and the drag ones. These phases are related to the angular position $\Theta$ of the control unit, with respect to the wind direction (see Figure 4).

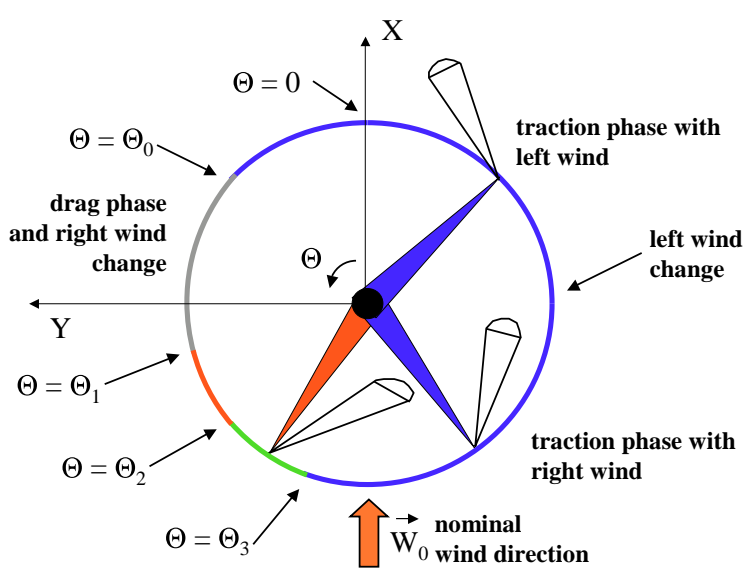

Fig. 4. Carousel configuration phases

During the traction phase, which begins at $\Theta=\Theta_{3}$ (see Figure 4), the control is designed in such a way that the kite pulls the rotor, maximizing the generated power. This phase ends at $\Theta=\Theta_{0}$ and the drag phase begins: the kite is no more able to generate a positive power until angle $\Theta$ reaches the value $\Theta_{3}$. In this phase, the control is designed to move the kite, with as low energy loss as possible, in the suitable position to begin another traction phase, where once again the control is designed to maximize the generated power. The control design is here carried on using a Fast implementation of a Predictive Controller (FMPC) as proposed in [9] and [10] and used also in [4] for the yo-yo configuration. Indeed, in each phase the design is formulated as an optimization problem with its own cost function, aimed to maximize the overall generated power, with state and input constraints, since for example the kite height on the ground cannot be negative and control actuators have their own physical limits. From this point of view, Model Predictive Control (MPC) appears to be an appropriate technique. However, a "fast" implementation is needed for the real time control computations at the required sampling time (of the order of $0.1 \mathrm{~s})$.

\section{KITE GENERATOR MODEL}

In this paper, a single arm rotor generator is considered. The kite control unit is located at the end of the rotor arm, whose length is indicated with $R$ in Figure 5. A fixed cartesian coordinate system $(X, Y, Z)$ is considered, with the origin located at the rotor center and $X$ axis aligned with the nominal wind speed vector direction. Rotation of the generator rotor around $Z$ axis is given by angle $\Theta$ as depicted in Figures 4 and 5. Wind speed vector is represented 
as $\vec{W}_{l}=\vec{W}_{0}+\vec{W}_{t}$, where $\vec{W}_{0}$ is the nominal wind, supposed to be known and expressed in $(X, Y, Z)$ as:

$$
\vec{W}_{0}=\left(\begin{array}{c}
W_{x}(Z) \\
0 \\
0
\end{array}\right)
$$

$W_{x}(Z)$ is a known function that returns the wind nominal speed at a certain height $Z$. The term $\vec{W}_{t}$ may have components in all directions and is not supposed to be known, accounting for wind unmeasured turbulence.

A moving cartesian system $(A, B, C)$ is also considered, centered at the end of the rotor arm and fixed to it. System $(A, B, C)$ is aligned with $(X, Y, Z)$ when $\Theta=0$. The position of the kite in $(A, B, C)$ can be expressed as a function of its distance $r$ from the origin and of the two angles $\theta$ and $\phi$, as depicted in Figure 5, which also shows the three basis vectors $e_{\theta}, e_{\phi}$ and $e_{r}$ of a local coordinate system, centered in the kite center of gravity. These basis vectors are expressed in the moving cartesian system $(A, B, C)$ by:

$$
\left(\begin{array}{lll}
\left(\begin{array}{lll}
e_{\theta} & e_{\phi} & e_{r}
\end{array}\right)= \\
\cos (\theta) \cos (\phi) & -\sin (\phi) & \sin (\theta) \cos (\phi) \\
\cos (\theta) \sin (\phi) & \cos (\phi) & \sin (\theta) \sin (\phi) \\
-\sin (\theta) & 0 & \cos (\theta)
\end{array}\right)
$$

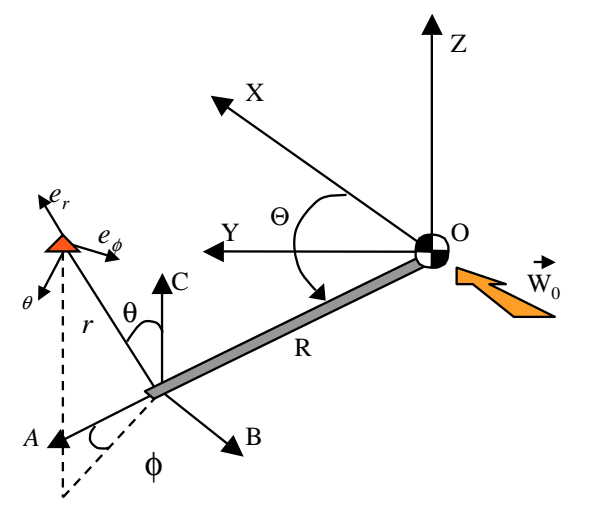

Fig. 5. Kite generator model diagram

The generator rotor motion law is given by the following equation:

$$
J_{z} \ddot{\Theta}=R F^{c} \sin \theta \sin \phi-T^{\mathrm{gen}}
$$

where $J_{z}$ is the rotor moment of inertia, $F^{c}$ is the pulling force exerted by the kite on its lines and $T^{\text {gen }}$ is the torque given by the electric generator linked to the rotor. Note that any viscous term is neglected in equation (3), since the rotor speed $\dot{\Theta}$ is kept very low as it will be shown in Section IV. Force $F^{c}$ is always directed along the local unit vector $e_{r}$ and cannot be negative, since the kite can only pull the lines. $T^{\text {gen }}$ is positive when the kite is pulling the rotor toward increasing $\Theta$ values, thus generating energy, and it is negative when the electric generator is acting as a motor to drag the rotor between $\Theta_{0}$ and $\Theta_{3}$ during the drag phase as depicted in Figure 4. Force $F_{c}$ is calculated by the kite control unit in order to keep the length of the lines constant (i.e. $r=r_{0}$ ), apart from the limited movements needed to control the kite, while torque $T^{\text {gen }}$ is calculated by a suitable local controller in order to keep the rotor at constant speed $\dot{\Theta}=\dot{\Theta}_{\text {ref }}$.

The kite dynamics are described by the model originally developed in [5]. Applying Newton's laws of motion to the kite in the local coordinate system and considering that the length of the lines is kept constant, the following three equations are obtained:

$$
\begin{aligned}
& r_{0} \ddot{\theta}=\frac{F_{\theta}}{m} \\
& r_{0} \sin (\theta) \ddot{\phi}=\frac{F_{\phi}}{m} \\
& \frac{F_{r}}{m}=0
\end{aligned}
$$

where $m$ is the kite mass. Forces $F_{\theta}, F_{\phi}$ and $F_{r}$ include the contributions of gravitational force $m g$, apparent force $\vec{F}^{\text {app }}$, aerodynamic force $\vec{F}^{\text {aer }}$ and force $F^{c}$ exerted by the kite on the lines. Their relations, expressed in the local coordinates, are given by:

$$
\begin{aligned}
& F_{\theta}=\sin (\theta) m g+F_{\theta}^{\mathrm{app}}+F_{\theta}^{\mathrm{aer}} \\
& F_{\phi}=F_{\phi}^{\mathrm{app}}+F_{\phi}^{\mathrm{aer}} \\
& F_{r}=-\cos (\theta) m g+F_{r}^{\mathrm{app}}+F_{r}^{\mathrm{aer}}-F^{c}
\end{aligned}
$$

$\vec{F}^{\text {app }}$ components in the local system are given by the following equations:

$$
\begin{array}{r}
F_{\theta}^{\text {app }}=m\left(\dot{\Theta}^{2} R \cos \theta \cos \phi-\ddot{\Theta} R \cos \theta \sin \phi\right. \\
\left.+(\dot{\Theta}+\dot{\phi})^{2} r_{0} \sin \theta \cos \theta\right) \\
F_{\phi}^{\text {app }}=m\left(-\ddot{\Theta} r_{0} \sin \theta-2(\dot{\Theta}+\dot{\phi}) \dot{\theta} r_{0} \cos \theta\right. \\
\left.-\ddot{\Theta} R \cos \phi-\dot{\Theta}^{2} R \sin \phi\right) \\
F_{r}^{\text {app }}=m\left(r_{0} \dot{\theta}^{2}+r_{0}(\dot{\Theta}+\dot{\phi})^{2} \sin ^{2} \theta-\ddot{\Theta} R \sin \theta \sin \phi\right. \\
\left.+\dot{\Theta}^{2} R \sin \theta \cos \phi\right)
\end{array}
$$

$\vec{F}^{\text {aer }}$ depends on the effective wind speed $\vec{W}_{e}$, which in the local system is calculated as:

$$
\vec{W}_{e}=\vec{W}_{a}-\vec{W}_{l}
$$

$\vec{W}_{a}$ is the kite speed, expressed in the local system as:

$$
\vec{W}_{a}=\left(\begin{array}{c}
\dot{\theta} r_{0}+\dot{\Theta} \cos \theta \sin \phi R \\
(\dot{\phi}+\dot{\Theta}) r_{0} \sin \theta+\dot{\Theta} \cos \phi R \\
\dot{\Theta} \sin \theta \sin \phi R
\end{array}\right)
$$

Let us consider now the kite wind axis system, with the origin in the kite center of mass, $\vec{x}_{\mathrm{w}}$ basis vector aligned with the effective wind speed vector, $\vec{z}_{\mathrm{w}}$ basis vector contained by the kite longitudinal mirror symmetry plane and pointing from the top surface of the kite to the bottom, and wind $\vec{y}_{\mathrm{w}}$ basis vector completing the right handed system. In the wind system the aerodynamic force $\vec{F}_{w}^{\text {aer }}$ is given by:

$$
\vec{F}_{w}^{\text {aer }}=F_{D} \vec{x}_{\mathrm{w}}+F_{L} \vec{z}_{\mathrm{w}}
$$

where $F_{D}$ is the drag force and $F_{L}$ is the lift force, calculated as:

$$
\begin{aligned}
& F_{D}=-\frac{1}{2} C_{D} A \rho\left|W_{e}\right|^{2} \\
& F_{L}=-\frac{1}{2} C_{L} A \rho\left|W_{e}\right|^{2}
\end{aligned}
$$

where $\rho$ is the air density, $A$ is the kite characteristic area, $C_{L}$ and $C_{D}$ are the kite lift and drag coefficients. All of 
these variables are supposed to be constant. $\vec{F}^{\text {aer }}$ can then be expressed in the local coordinate system as a nonlinear function of several arguments:

$$
F^{\mathrm{aer}}=\left(\begin{array}{c}
F_{\theta}^{\mathrm{aer}}\left(\theta, \phi, \Theta, \psi, \vec{W}_{e}\right) \\
F_{\phi}^{\mathrm{aer}}\left(\theta, \phi, \Theta, \psi, \vec{W}_{e}\right) \\
F_{r}^{\mathrm{aer}}\left(\theta, \phi, \Theta, \psi, \vec{W}_{e}\right)
\end{array}\right)
$$

Angle $\psi$ indicated in (11) is the control variable, defined by

$$
\psi=\arcsin \left(\frac{\Delta l}{d}\right)
$$

with $d$ being the distance between the two lines fixing points at the kite and $\Delta l$ the length difference of the two lines. Angle $\psi$ influences the kite motion by changing the direction of the vector $\vec{F}$ aer .

Thus the system dynamics are of the form:

$$
\dot{x}(t)=g\left(x(t), u(t), \vec{W}_{l}(t)\right)
$$

where $x(t)=[\theta(t) \phi(t) \Theta(t) \dot{\theta}(t) \dot{\phi}(t) \dot{\Theta}(t)]^{T}$ and $u(t)=$ $\psi(t)$. All the model states are supposed to be measured, to be used for feedback control.

\section{Kite CONTROL USING MPC}

Control problem and related objectives are now described. As highlighted in the Introduction, the main objective is to generate energy by a suitable control action on the kite. In order to accomplish this aim, a two-phase cycle has been defined. The two phases are referred to as the traction phase and the drag phase. In both phases, MPC controllers are designed, according to their own functional, state and input constraints and terminal conditions.

The control move computation is performed at discrete time instants defined on the basis of a suitably chosen sampling period $\Delta_{t}$. At each sampling time $t_{k}=k \Delta_{t}, k \in \mathbb{Z}^{+}$, the measured values of the state $x\left(t_{k}\right)$ and of the nominal wind speed $W_{x}\left(t_{k}\right)$ are used to compute the control move through the optimization of a performance index of the form:

$$
J\left(U, t_{k}, T_{p}\right)=\int_{t_{k}}^{t_{k}+T_{p}} L\left(\tilde{x}(\tau), \tilde{u}(\tau), W_{x}(\tau),\right) d \tau
$$

where $T_{p}=N_{p} \Delta_{t}, N_{p} \in \mathbb{Z}^{+}$is the prediction horizon, $\tilde{x}(\tau)$ is the state predicted inside the prediction horizon according to the state equation (13), using $\tilde{x}\left(t_{k}\right)=x\left(t_{k}\right)$ and the piecewise constant control input $\tilde{u}(t)$ belonging to the sequence $U=\{\tilde{u}(t)\}, t \in\left[t_{k}, t_{k+T_{p}}\right]$ defined as:

$\tilde{u}(t)=\left\{\begin{array}{l}\bar{u}_{i}, \forall t \in\left[t_{i}, t_{i+1}\right], i=k, \ldots, k+T_{c}-1 \\ \bar{u}_{k+T_{c}-1}, \forall t \in\left[t_{i}, t_{i+1}\right], i=k+T_{c}, \ldots, k+T_{p}-1\end{array}\right.$

where $T_{c}=N_{c} \Delta_{t}, N_{c} \in \mathbb{Z}^{+}, N_{c} \leq N_{p}$ is the control horizon.

The function $L(\cdot)$ in (14) is suitably defined on the basis of the performances to be achieved in the operating phase the kite generator lies in. Moreover, in order to take into account physical limitations on both the kite behaviour and the control input $\psi$ in the different phases, linear constraints of the form $F \tilde{x}(t)+G \tilde{u}(t) \leq H$ have been included too.
Thus the predictive control law is computed using a receding horizon strategy:

1) At time instant $t_{k}$, get $x\left(t_{k}\right)$.

2) Solve the optimization problem:

$$
\begin{gathered}
\min _{U} \quad J\left(U, t_{k}, T_{p}\right) \\
\text { subject to } \\
\dot{\tilde{x}}(t)=g\left(\tilde{x}(t), \tilde{u}(t), W_{x}(t)\right) \\
F \tilde{x}(t)+G \tilde{u}(t) \leq H, \forall t \in\left[t_{k}, t_{k+T_{p}}\right]
\end{gathered}
$$

3) Apply the first element of the solution sequence $U$ to the optimization problem as the actual control action $u\left(t_{k}\right)=\tilde{u}\left(t_{k}\right)$.

4) Repeat the whole procedure at the next sampling time $t_{k+1}$.

Therefore the predictive controller results to be a nonlinear static function of the system state $x$ and the nominal measured wind speed $W_{x}$ :

$$
\psi\left(t_{k}\right)=f\left(x\left(t_{k}\right), W_{x}\left(t_{k}\right), \dot{\Theta}_{\text {ref }}\left(t_{k}\right)\right)
$$

A. Traction phase

The aim of this phase is to obtain as much mechanical energy as possible from the wind stream. The traction phase begins when the rotor angular position $\Theta$ with respect to the nominal wind vector $\vec{W}_{0}$ is such that the kite can pull the rotor arm (see Figure 4). Thus, the following traction phase initial condition is considered:

$$
\Theta(t) \geq \Theta_{3}
$$

Control system objective adopted in the traction phase is to maximize the energy generated in the interval $\left[t_{k}, t_{k}+T_{P}\right]$, while satisfying constraints concerning state and input values. Mechanical power generated at each instant is $P=$ $\dot{\Theta} T^{\text {gen }}$, thus the following cost function is chosen to be minimized in MPC design (16):

$$
J\left(t_{k}\right)=-\int_{t_{k}}^{t_{k}+T_{p}}\left(\dot{\Theta}(\tau) T^{\mathrm{gen}}(\tau)\right) d \tau
$$

During the whole phase the following state constraint is considered to keep the kite sufficiently far from the ground:

$$
\theta(t) \leq \bar{\theta}
$$

with $\bar{\theta}<\pi / 2$. Actuator physical limitations give rise to the constraints:

$$
\begin{aligned}
& |\psi(t)| \leq \bar{\psi} \\
& |\dot{\psi}(t)| \leq \bar{\psi}
\end{aligned}
$$

As a matter of fact, other technical constraints have been added to force the kite to go along "lying eight" trajectories rather than circular ones, in order to prevent the winding of the lines. Such constraints force the kite $\phi$ angle to oscillate with double period with respect to $\theta$ angle, thus generating the proper kite trajectory.

The traction phase ends when the rotor angle is such that the kite is no more able to pull the rotor arm:

$$
\Theta(t) \geq \Theta_{0}
$$

with $\Theta_{0} \leq \pi / 2$ according to Figure 4 . When the condition (22) is reached the drag phase can start. 


\section{B. Drag phase}

During this phase the electric generator act as a motor to drag the rotor between angles $\Theta_{0}$ and $\Theta_{3}$. Meanwhile, the kite is moved in a proper position in order to start another traction phase. The drag phase has been divided into three sub-phases. Transitions between each two subsequent drag sub-phases are marked by suitable values of the rotor angle, $\Theta_{1}$ and $\Theta_{2}$, which are chosen in order to minimize the total energy spent during the phase.

In the first sub-phase, the control objective is to move the kite in a zone with low values of $\theta$, where effective wind speed $\vec{W}_{e}$ and pulling force $F^{c}$ component in plane $(X, Y)$ (i.e. $F^{c} \sin \theta \sin \phi$ ) are much lower. A positive value $\theta_{I}<\pi / 2$ of $\theta$ is introduced to identify this zone. The following cost function is considered:

$$
J\left(t_{k}\right)=\int_{t_{k}}^{t_{k}+T_{p}}\left(\theta(\tau)-\theta_{I}\right)^{2} d \tau
$$

Once the following condition is reached:

$$
\Theta \geq \Theta_{1}
$$

the first drag phase part ends.

In the second drag sub-phase, control objective is to change the kite angular position $\phi$ toward values which are suitable to begin the traction phase. Thus, value $\phi_{I}$ is introduced such that

$$
\pi / 2<\phi_{I}<3 \pi / 2
$$

and the following cost function is considered:

$$
J\left(t_{k}\right)=\int_{t_{k}}^{t_{k}+T_{p}}\left(\phi(\tau)-\phi_{I}\right)^{2} d \tau
$$

The second sub-phase ends when the following condition is satisfied:

$$
\Theta \geq \Theta_{2}
$$

Then, the third drag sub-phase begins: control objective is to increase the kite angle $\theta$ toward a suitable value $\theta_{I I}$ such that:

$$
\pi / 4<\theta_{I I}<\pi / 2
$$

thus preparing the generator for the following traction phase. Cost function $J\left(t_{k}\right)$ is set as follows:

$$
J\left(t_{k}\right)=\int_{t_{k}}^{t_{k}+T_{p}}\left(\theta(\tau)-\theta_{I I}\right)^{2} d \tau
$$

Ending conditions for the whole drag phase coincide with starting conditions for the traction phase (18).

During the whole drag phase the state constraint expressed by (20) and the input constraints (21) are considered in the control optimization problems.

For any of the MPC controller previously described, control $\psi\left(t_{k}\right)$ results to be the nonlinear static function given by (17), which can be rewritten as:

$$
\psi\left(t_{k}\right)=f\left(w\left(t_{k}\right)\right)
$$

Where $w\left(t_{k}\right)=\left(x\left(t_{k}\right), W_{x}\left(t_{k}\right), \dot{\Theta}_{\text {ref }}\left(t_{k}\right)\right)^{T}$. For a given $w\left(t_{k}\right)$, the value of the function $f\left(w\left(t_{k}\right)\right)$ is typically computed by solving at each sampling time $t_{k}$ the constrained optimization problem (16). However, an online solution of the optimization problem at each sampling time cannot be performed at the sampling period required for this application, of the order of $0.1 \mathrm{~s}$. An approach to overcome this problem is to evaluate off line a certain number of values of $f(w)$ to be used to find an approximation $\hat{f}$ of $f$, suitable to be used for online implementation. In particular the FMPC approach introduced and described in [9] [10] and also used in [4] based on Set Membership approximation techniques will be employed to derive the approximating function $\hat{f}$.

\section{Simulation Results}

Simulations have been performed with the values of model and control parameters reported in Table I. Table II contains the state values which identify each phase starting and ending conditions and the values of state and input constraints.

TABLE I

MODEL AND CONTROL PARAMETERS

\begin{tabular}{|l|r|l|}
\hline$m$ & 50 & kite mass $(\mathrm{kg})$ \\
\hline$A$ & 100 & characteristic area $\left(\mathrm{m}^{2}\right)$ \\
\hline$r_{0}$ & 300 & line length $(\mathrm{m})$ \\
\hline$J_{z}$ & $910^{8}$ & rotor moment of inertia $\left(\mathrm{kg} \mathrm{m}^{2}\right)$ \\
\hline$R$ & 300 & rotor radius $(\mathrm{m})$ \\
\hline$\rho$ & 1.2 & air density $\left(\mathrm{kg} / \mathrm{m}^{3}\right)$ \\
\hline$C_{L}$ & 1.2 & lift coefficient \\
\hline$C_{D}$ & 0.15 & drag coefficient \\
\hline$\dot{\Theta}_{\text {ref }}$ & 0.16 & reference $\dot{\Theta}(\mathrm{rpm})$ \\
\hline$T_{c}$ & 0.2 & sample time $(\mathrm{s})$ \\
\hline$N_{c}$ & 1 & control horizon (steps) \\
\hline$N_{p}$ & 8 & prediction horizon (steps) \\
\hline
\end{tabular}

TABLE II

CYCLE PHASES OBJECTIVES AND STARTING CONDITIONS, STATE AND INPUT CONSTRAINTS

\begin{tabular}{|l|r|l|}
\hline$\Theta_{0}$ & $45(\mathrm{deg})$ & Drag phase starting condition \\
\hline$\theta_{I}$ & $20(\mathrm{deg})$ & $1^{\text {st }}$ Drag sub-phase objective \\
\hline$\Theta_{1}$ & $135(\mathrm{deg})$ & $2^{\text {nd }}$ Drag sub-phase starting condition \\
\hline$\phi_{I}$ & $140(\mathrm{deg})$ & $2^{\text {nd }}$ Drag sub-phase objective \\
\hline$\Theta_{2}$ & $150(\mathrm{deg})$ & $3^{\text {rd }}$ Drag sub-phase starting condition \\
\hline$\theta_{I I}$ & $50(\mathrm{deg})$ & $3^{\text {rd }}$ Drag sub-phase objective \\
\hline$\Theta_{3}$ & $165(\mathrm{deg})$ & Traction phase starting condition \\
\hline $\bar{\theta}$ & $85(\mathrm{deg})$ & State constraint \\
\hline $\bar{\psi}$ & $3(\mathrm{deg})$ & Input constraints \\
\hline $\bar{\psi}$ & $20(\mathrm{deg} / \mathrm{s})$ & \\
\hline
\end{tabular}

Note that the reference rotor speed $\dot{\Theta}_{\text {ref }}$ is very low, according to the hypothesis of Section II. The first component of the nominal wind speed, according to (1), is given as:

$W_{x}(Z)= \begin{cases}0.04 Z+8 & \text { if } Z \leq 100 \mathrm{~m} \\ 0.0172(Z-100)+12 & \text { if } Z>100 \mathrm{~m} / \mathrm{s}\end{cases}$

Nominal wind speed is $8 \mathrm{~m} / \mathrm{s}$ at $0 \mathrm{~m}$ of height and grows linearly to $12 \mathrm{~m} / \mathrm{s}$ at $100 \mathrm{~m}$ and up to $17 \mathrm{~m} / \mathrm{s}$ at $300 \mathrm{~m}$ of height. In the first simulation, no wind turbulence was considered, so $W_{t}(t)=0$. In the second simulation the following sinusoidal wind turbulence was introduced along the fixed $Y$ axis:

$$
W_{t}(t)=2.5 \sin \left(\omega_{0} t\right) \mathrm{m} / \mathrm{s}
$$


with $\omega_{0}=2 \pi / 80 \mathrm{rad} / \mathrm{s}$. Finally, in the third simulation the same wind turbulence was introduced along the fixed vertical axis $Z$. Note that the amplitude of $W_{t}$ is higher than $20 \%$ of nominal wind speed at 100 meters of height, thus introducing strong disturbances in the system.

Figure 6 shows the trajectories of the kite and of the control unit during two full cycles in nominal conditions. Figure 7 depicts some orbits traced by the kite during the traction phase: it can be seen that the kite follows "lying eight" orbits in this phase, with a period of about $4 \mathrm{~s}$; about 65 orbits are thus completed in a single traction phase. The power generated during the two cycles is reported in Figure 8: the mean value is $478 \mathrm{~kW}$ and the consequent generated energy is $180 \mathrm{MJ}$ per cycle. Figure 9 depicts the behaviour of the wind effective speed magnitude $\left|\vec{W}_{e}\right|$ during two full cycles. It can be noted that during the traction phase the kite speed is about 15 times greater than the rotor tangential speed, which is equal to $18 \mathrm{~km} / \mathrm{h}$ : this is one of the main advantages of KiteGen over classical wind mills, which work with much lower wind effective speed values.

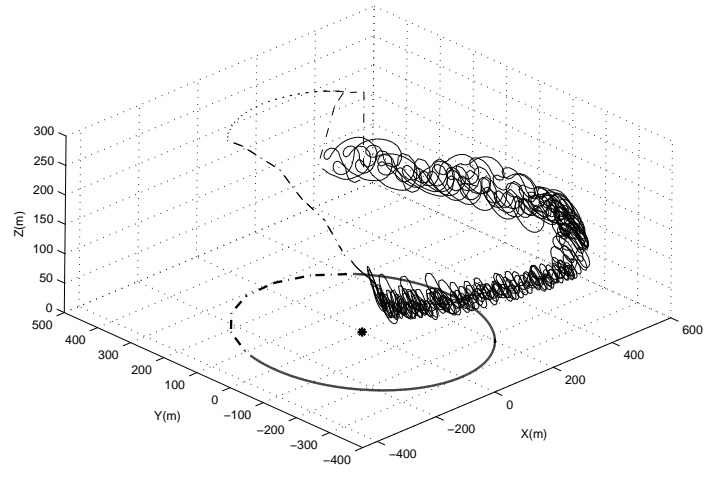

Fig. 6. Kite (thin line) and control unit trajectory with nominal conditions: traction phase (solid) and drag phase (dot-dash)

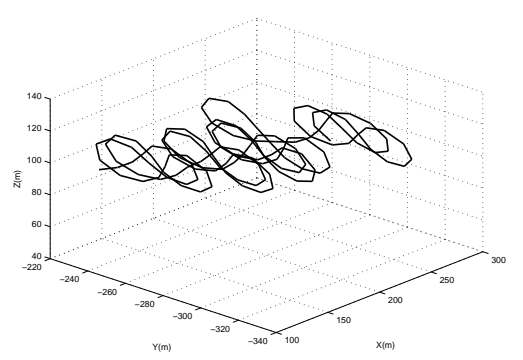

Fig. 7. Some traction phase orbits

Note that since the fixed coordinate system $(X, Y, Z)$ has been defined on the basis of the nominal wind direction, a measurable change of the latter can be easily overcome by rotating the whole coordinate system $(X, Y, Z)$, thus obtaining the same performances without changing neither the control system parameters nor the starting conditions of the various phases.

As regards the control system performances in presence of

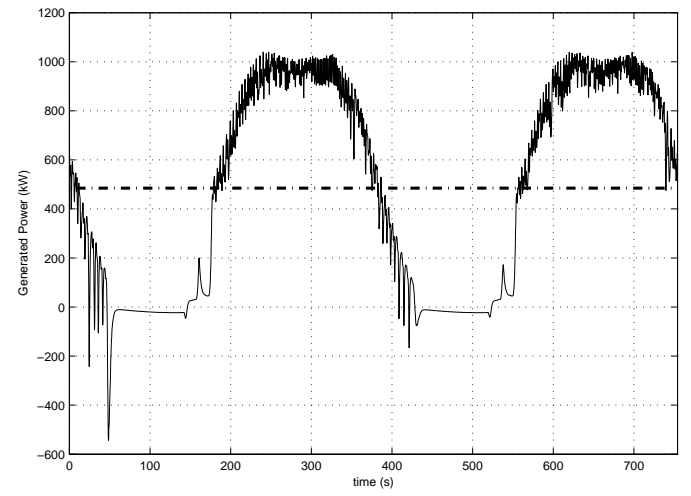

Fig. 8. Instant (solid) and mean (dashed) power generated during two cycles, nominal conditions

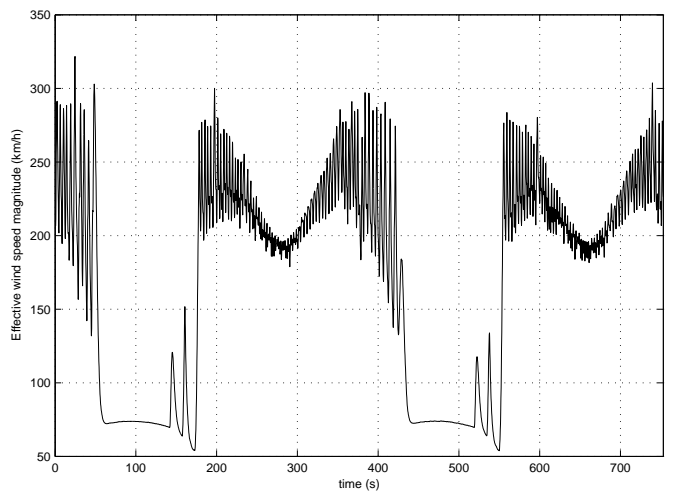

Fig. 9. Effective wind speed magnitude $\left|\vec{W}_{e}\right|$, nominal conditions

unmeasured wind changes, in Figure 10 the power generation results with the lateral wind disturbances described by (31) are shown: the cycles were completed and the mean generated power value, $475 \mathrm{~kW}$, is similar to the one obtained without disturbances, showing the good tolerance of the control system to lateral wind turbulence.

The simulated power generation behaviour in presence of the vertical turbulence described by (31) is reported in Figure 11: the cycles were completed with a mean generated power value of $485 \mathrm{~kW}$, showing good system robustness also in presence of severe vertical wind disturbances.

\section{CONClusions AND FUtuRE DEVElopments}

The paper has presented a study aimed to investigate the capability of controlling tethered airfoils in order to devise a new class of wind energy generators able to overcome the main limitations of the present aeolian technology based on wind mills. A single kite carousel configuration has been considered and the obtained results appear to be very encouraging, even though they are based on simulations carried on a kite model taken from the literature, which certainly can give only approximate description of involved dynamics. Indeed, accurate modelling the dynamic of non rigid airfoils is well known to be a challenging task and it can be expected that the control design based on the kite model considered in this paper may not perform in a satisfactory 


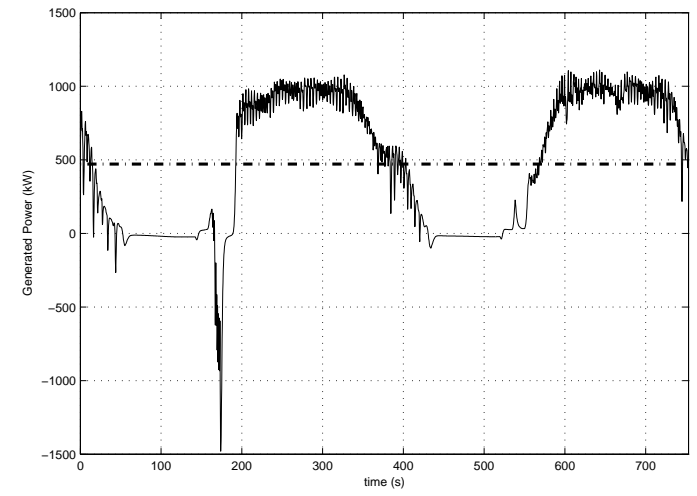

Fig. 10. Instant (solid) and mean (dashed) power generated during two cycles, lateral wind turbulence

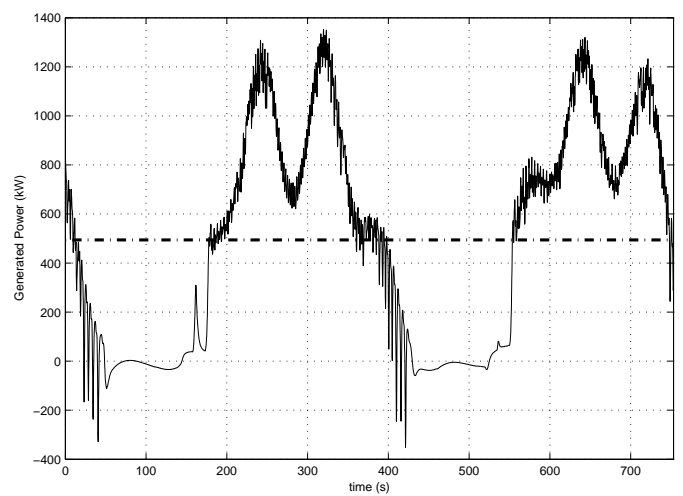

Fig. 11. Instant (solid) and mean (dashed) power generated during two cycles, vertical wind turbulence

way on the built prototype. Therefore, experimental data taken from the prototype will be employed, together with advanced methods for the identification of complex nonlinear systems such as [11], [12], in order to derive more accurate kite models, sufficient to obtain good performances from the NMPC design on the real application. On the other hand, the first tests performed on the built prototype in the yoyo configuration introduced in [4] show a good matching between simulations and experimental results as regards the generated power (as reported in [13]). Therefore, the considered model equations provide a good estimate of the power that can be obtained with kite generators, making it possible to draw conclusions about their scalability. In particular, a single $500 \mathrm{~m}^{2}$ kite with $12 \mathrm{~m} / \mathrm{s}$ nominal wind speed and aerodynamic efficiency (i.e. $C_{L} / C_{D}$ ) equal to 12 would be able to generate $10 \mathrm{MW}$ mean power. 100 such kites towing a $1500 \mathrm{~m}$ radius carousel would generate $1000 \mathrm{MW}$ mean power with about $7-8 \mathrm{~km}^{2}$ land occupation and with an estimated energy production cost ten times lower than the one obtained by fossil fuel thermal plants. Note that a wind farm producing the same mean power, using the present wind mill technology, would have a territory occupation of about $250-300 \mathrm{~km}^{2}$ and an energy production cost $40-50 \%$ higher than thermal plants. Thus, the presented results show that KiteGen could provide cheap renewable energy, in large quantity and with quite low territory occupation, opening the way to much larger contributions to the world electric energy demand than expected from the present renewable energy technologies.

\section{REFERENCES}

[1] Worldwatch Institute,"Renewables 2005: Global Status Report" prepared for the Renewable Energy Policy Network, Washington DC, 2005. http://www.REN21.net

[2] "Smart control system exploiting the characteristics of generic kites or airfoils to convert energy", European patent $\sharp 02840646$, inventor: M. Ippolito, December 2004

[3] "Sistema e procedimento di controllo automatico del volo di profili alari di potenza", Patent n. TO2006A000372, inventors: M. Milanese, M. Ippolito, May 2006

[4] M. Canale, L. Fagiano, M. Ippolito and M. Milanese, "Control of tethered airfoils for a new class of wind energy generator", in $45^{\text {th }}$ IEEE Conference on Decision and Control, San Diego (CA), USA, 2006.

[5] M. Diehl, "Real-Time Optimization for Large Scale Nonlinear Processes", PhD thesis, University of Heidelberg, Germany, 2001.

[6] B. Houska and M. Diehl, "Optimal Control for Power Generating Kites", in $9^{\text {th }}$ European Control Conference, Kos, Greece, 2007.

[7] B. Houska and M. Diehl, "Optimal Control of Towing Kites", in $45^{\text {th }}$ IEEE Conference on Decision and Control, San Diego (CA), USA, 2006.

[8] T. Parisini and R. Zoppoli, "A receding-horizon regulator for nonlinear systems and a neural approximation". Automatica 31(10), 1443-1451, 1995.

[9] M. Canale and M. Milanese, "FMPC: a fast implementation of model predictive control", in $16^{\text {th }}$ IFAC World Congress, Prague, Czech Republic, July 2005.

[10] M.Canale, L. Fagiano, M. Milanese, "Fast implementation of nonlinear model predictive controllers". Technical Report CaFM-1-2006. Dipartimento di Automatica e Informatica, Politecnico di Torino, 2006.

[11] M. Milanese and C. Novara, "Set membership identification of nonlinear systems", Automatica, vol. 40, pp. 957-975, 2004.

[12] M. Milanese, C. Novara and L. Pivano, "Structured Experimental Modelling of Complex Nonlinear Systems", $42^{\text {nd }}$ IEEE Conference on Decision and Control, Maui, Hawaii, 2003.

[13] KiteGen report $\sharp 3$, Dip. Automatica e Informatica, Politecnico di Torino, Italy, October 2006 